\title{
EFECTOS HETERÓTICOS Y APTITUD COMBINATORIA EN POBLACIONES DE CHILE DULCE (Capsicum annuum L.)
}

\section{COMBINING ABILITY AND HETEROTIC EFFECTS IN SWEET PEPPER POPULATIONS (Capsicum annuum L.)}

\author{
Anastacio M. Pech May ${ }^{1}$, Guillermo Castañón Nájera², José M. Tun Suárez ${ }^{1}$, Mariano Mendoza Elos ${ }^{3}$, \\ Javier O. Mijangos Cortés ${ }^{4}$, Alfonzo Pérez Gutiérrez ${ }^{1}$ y Luis Latournerie Moreno ${ }^{1 *}$
}

\begin{abstract}
${ }^{1}$ División de Estudios de Posgrado e Investigación, Instituto Tecnológico de Conkal. Km 16. 3 antigua carretera Mérida-Motul. 97345, Conkal, Yucatán, México. Tel: (999) 912-4130 ext. 139. ${ }^{2}$ División Académica de Ciencias Biológicas, Universidad Juárez Autónoma de Tabasco. km 0.5 carretera Villahermosa-Cárdenas. 86000, Villahermosa, Tabasco, México. ${ }^{3}$ Instituto Tecnológico de Roque. Celaya, Guanajuato, México. ${ }^{4}$ Unidad de Recursos Naturales, Centro de Investigación Científica de Yucatán. Calle 43 No. 130, Col. Chuburná de Hidalgo. 97200, Mérida, Yucatán, México.
\end{abstract}

* Autor para correspondencia (sayilhahil@yahoo.com.mx)

\section{RESUMEN}

Se estimó la aptitud combinatoria y la heterosis de siete poblaciones criollas de chile 'Dulce' (Capsicum annuum L.) y las cruzas resultantes entre ellas, mediante un diseño dialélico. Las 21 cruzas y sus progenitores se evaluaron en Akil y Acanceh, del Estado de Yucatán, México. La unidad experimental fue un surco de $8.0 \mathrm{~m}$ con 21 plantas distanciadas a $0.40 \mathrm{~m}$, con distancia entre surcos de $1.20 \mathrm{~m}$. Los experimentos se establecieron en un diseño bloques completos al azar con tres repeticiones. Se midió: rendimiento de fruto, peso individual de frutos, número de frutos por planta, días a inicio de cosecha, altura de planta, longitud y diámetro de fruto. Las aptitudes combinatorias general (ACG) y específica (ACE) se estimaron con el método II modelo I de Griffing. Se encontró que los efectos aditivos estimados por la ACG fueron más grandes que los de dominancia estimados por la ACE, y que ambos efectos fueron influenciados por el ambiente de evaluación. Tres progenitores (P2, P3 y P4) mostraron los efectos positivos más altos de ACG en el rendimiento de fruto, $y$ dos de éstos generaron híbridos con altos valores de ACE y heterosis. Por los valores de ACG de los padres y de heterosis de las progenies, se concluye que la hibridación sería el método de mejoramiento genético más adecuado para incrementar rendimiento de fruto y número de frutos por planta. En cambio, para mejorar altura de planta, peso individual de fruto, días a inicio de cosecha, longitud y diámetro de fruto, el método de mejoramiento por endocría y selección sería el indicado, para formar variedades.

Palabras clave: Capsicum annuum, dialélico, variedades criollas, habilidad combinatoria, heterosis.

\section{SUMMARY}

Combining ability and heterosis of seven sweet pepper landraces (Capsicum annuum $\mathbf{L}$.) and their crosses were estimated in a diallel mating design. The 21 crosses and their parents were evaluated in Akil and Acanceh, locations in the state of Yucatán, México. The experimental unit was a single row of $8.0 \mathrm{~m}$ long with 21 plants; distance between plants was $0.40 \mathrm{~m}$ and $1.20 \mathrm{~m}$ between rows. The experiments were established in a randomized complete block design with three replications. Total fruit yield, single fruit weight, number of fruits per plant, days to first harvest, plant height, fruit length and fruit diameter were measured. The general combining ability (GCA) and the specific combining ability (SCA) were estimated using Method II Model I by Griffing. Results showed that additive effects estimated by GCA were higher than the dominance effects estimated by SCA, and both effects were highly influenced by environment. Three parents (P2, P3 and P4) showed the highest positive GCA effects on fruit yield; two of them inherited to their hybrids high values of SCA and heterosis. Because of the GCA high values observed in parents and the high heterosis in progenies, it is concluded that hybridization would be the best breeding method for increasing total fruit yield and number of fruits per plant. However, for improving plant height, individual fruit weight, days to first harvest fruit length, and fruit diameter the proper breeding method should be by inbreeding and selection.

Index words: Capsicum annuиm, diallel, landraces, heterosis, combining ability.

\section{INTRODUCCIÓN}

Heterosis es la expresión de un carácter en la progenie más allá de los límites de expresión manifestada en sus progenitores que tiene origen en los efectos genéticos principalmente de dominancia y en la diferencia genotípica de frecuencias génicas (Falconer, 1996). La heterosis ha sido ampliamente utilizada en programas de mejoramiento de muchos cultivos para la identificación de poblaciones genéticamente divergentes, como base para el desarrollo de líneas endogámicas a ser usadas en cruzamientos F1 (Hallauer y Miranda, 1988).

Diversos investigadores han reportado efectos de heterosis alta en Capsicum spp., para largo y diámetro de fruto, número de semillas por fruto, rendimiento y 
contenido de capsaicina por planta (De Souza y Maluf, 2003; Seneviratne y Kannangara, 2004), rendimiento y calidad de frutos (Milerue y Nikornpun, 2000; PérezGrajales et al., 2009), peso de semillas por fruto, peso de 100 semillas, número de frutos por planta (Mishra et al., 1989), contenidos de vitamina C y capsaicinoides en diferentes grados de madurez de fruto (Cruz-Pérez et al., 2007), materia seca de fruto por planta, incidencia de Xanthomonas (Blank y Maluf, 1997; De Souza y Maluf, 2003) y para contenido de capsaicina (Zewdie et al., 2000; Zewdie y Bosland, 2000). La mayoría de esos estudios se han realizado en $C$. annuum, y en pocas investigaciones se ha determinado la magnitud de heterosis en otras especies, como $C$. chinense y $C$. pubescens. En México existen pocos reportes de heterosis en chile.

Las cruzas dialélicas han sido usadas para investigar la herencia de importantes características. Específicamente, las cruzas dialélicas fueron diseñadas para investigar la aptitud combinatoria general (ACG) de los progenitores y para identificar los progenitores superiores a ser usados en el desarrollo de híbridos y cultivares (Yan y Hunt, 2002). Según Sprague y Tatum (1942), la ACG corresponde al comportamiento promedio de una línea en diversas combinaciones híbridas y la aptitud combinatoria específica (ACE) a las combinaciones específicas con respecto a la ACG de sus padres. La teoría y la forma del análisis de cruzas dialélicas fue propuesta por Jinks y Hayman (1953) y Jinks (1954). Se han desarrollado varios métodos para el análisis de cruzas dialélicas, pero los más usados son los propuestos por Griffing (1956).

En chile se ha explotado la heterosis para incrementar el rendimiento y otros caracteres económicos (Seneviratne y Kannangara, 2004), y se considera que en Capsicum la heterosis es alta (De Souza y Maluf, 2003). Por ello, la existencia de una amplia diversidad de este género en México, tanto en el ámbito de variantes cultivadas como semicultivadas y silvestres, puede aprovecharse para formar híbridos locales y nacionales, ya que la semilla híbrida que se usa proviene de empresas trasnacionales. En la Península de Yucatán es importante aprovechar la diversidad de tipos de chiles, que son conservados y aprovechados por los productores. Entre los más importantes se encuentran el chile 'Habanero' ( $C$. chinense Jacq.) y los pertenecientes a $C$. annuum ('Dulce' e 'Xcat'ik') (Latournerie et al., 2001). El chile 'Dulce' se distingue porque no contiene capsaicina, y su fruto presenta formas que van desde redondas a ligeramente alargadas y arriñonadas (Pozo et al., 1991).

En Yucatán se siembran anualmente más de 200 ha de este tipo de chile criollo, tanto en condiciones de riego como de temporal o secano (SAGARPA, 2008). Además, es común encontrarlo sembrado en macetas caseras, para autoconsumo. En la región se ha realizado poco mejoramiento genético y se ha hecho solamente para el 'Habanero'. Es entonces necesario aplicar algún método de mejoramiento genético a las poblaciones criollas de chile 'Dulce' que se cultivan en este estado, para generar cultivares con mayor producción y calidad del fruto. En el presente trabajo se planteó el objetivo de estimar las aptitudes combinatorias general y específica, y la heterosis que puedan existir entre materiales de chile 'Dulce'.

\section{MATERIALES Y MÉTODOS}

Como material parental se usaron siete poblaciones criollas de chile 'Dulce' denominadas ITCD: 246(P1), 31(P2), 7(P3), 183(P4), 207(P5), 209(P6) у 210(P7), los cuales fueron seleccionadas con base en su rendimiento de fruto y comportamiento agronómico registrado en evaluaciones previas, de un total de 12. Las cruzas posibles se hicieron en forma manual en invernadero, a la vez que se incrementaba la semilla de los progenitores.

Para la evaluación de las 21 cruzas simples y los siete progenitores se estableció un experimento en un diseño bloques completos al azar con tres repeticiones. Las localidades de prueba fueron dos: 1) Akil $\left(20^{\circ} 14^{\prime}\right.$ y $20^{\circ}$ 22' LN y $89^{\circ} 18^{\prime}$ y $89^{\circ} 26^{\prime} \mathrm{LO}$, a $31 \mathrm{msnm}$ ), donde el experimento se condujo con riego por inundación en el periodo de junio a septiembre de 2005 (con temperatura media anual de $26.2{ }^{\circ} \mathrm{C}$ y precipitación media de 731 $\mathrm{mm})$; y 2 ) Acanceh $\left(20^{\circ} 48^{\prime}\right.$ y $20^{\circ} 56^{\prime} \mathrm{LN}$ y $89^{\circ} 23^{\prime}$ y $89^{\circ} 32^{\prime} \mathrm{LO}$, a $14 \mathrm{msnm}$ ), con riego presurizado en el periodo de abril a junio de 2006 (con temperatura media anual de $25{ }^{\circ} \mathrm{C}$ y precipitación media de $704 \mathrm{~mm}$ ). En ambas localidades el suelo es del tipo Rendzina que corresponde a los Kancab, con base en la clasificación maya (Duch, 1988). En Acanceh el suelo profundo contenía bagazo de henequén en descomposición avanzada $\mathrm{y}$ es menos pedregoso que Akil cuyo suelo es poco profundo.

Las plántulas se obtuvieron en invernadero, a partir de semillas que se sembraron en charolas de poliestireno con 200 cavidades rellenas con el sustrato comercial Cosmopeat ${ }^{\circledR}$. Cuando las plántulas alcanzaron una altura de $20 \mathrm{~cm}$ se trasplantaron en campo. La unidad experimental fue un surco de $8.0 \mathrm{~m}$ con 21 plantas. La distancia entre plantas fue $0.40 \mathrm{~m}$, y de $1.20 \mathrm{~m}$ entre hileras. El manejo del cultivo (fertilización, y control de plagas y enfermedades) se efectuó con base en el paquete tecnológico propuesto por Soria et al. (2000). 
Como variables de respuesta se midió: rendimiento total de fruto $(\mathrm{g} / \mathrm{planta})$, peso individual del fruto $(\mathrm{g})$, número de frutos por planta, días a inicio de cosecha (cuando los frutos adquirieron un color verde brillante y consistencia dura al tacto), altura de planta $(\mathrm{cm})$ medida después del último corte, longitud de fruto $(\mathrm{mm})$ y diámetro de fruto (mm).

La información de los caracteres evaluados se analizó en forma combinada a través de localidades mediante el método II (incluye progenitores y sus cruzas) modelo I (efectos fijos) de Griffing (1956). Se usó el algoritmo de análisis computarizado desarrollado por Castañón et al. (2005). El modelo base de análisis de varianza fue:

$$
Y_{i j k r}=\mu+l_{k}+r_{r(k)}+g_{i}+g_{j}+s_{i j}+g l_{i k}+g l_{j k}+s l_{i j k}+\varepsilon i j k r
$$

Donde: $Y_{i j k r}$ es el valor fenotípico observado de la cruza con los progenitores $i$ y $j$ en la r-ésima repetición en la localidad $k ; \mu$ es la media general de la población; $l_{k}$ es el efecto de la $k$-ésima localidad, $r_{r(k)}$ es el efecto de la $r$-ésima repetición de la $k$-ésima localidad; $g_{i}$ y $g_{j}$ son el efecto de ACG para el $i$-ésimo y j-ésimo progenitor; $s_{i j}$ es el efecto de ACE de la cruza entre el i-ésimo y el j-ésimo progenitor; $g l_{i k}$ es la interacción entre el efecto de ACG para el progenitor $i$ y localidad $k, g l_{j k}$ es la interacción entre el efecto de ACG del progenitor $j$ en la localidad $k$; $s l_{i j k}$ es la interacción entre el efecto de ACE para el ij-ésimo híbrido y la localidad $k$; y eijkr es el error asociado con $Y_{i j k r}$.

La comparación de medias se hizo con el criterio de Antuna et al. (2003), en el que los valores superiores de una característica en particular son los que superaron a la media general más dos veces su correspondiente error estándar $(\mu+2 \sigma)$.

La heterosis se estimó como la diferencia entre el promedio de una cruza en relación con el promedio de sus progenitores, expresada en términos porcentuales:

$$
H=\frac{\bar{F}_{l i j}-\bar{P}_{l i j}}{P_{l i j}^{-}} \times 100
$$

Donde: $\bar{F}_{l i j}$ es la media del híbrido entre los padres $P_{i} \mathrm{y}$ $P_{j} ; \bar{P}_{l i j}$ es la media de los padres $P_{i}$ y $P_{j}$ para la $i j$-ésima cruza. La significancia de la heterosis $(H)$ fue determinada mediante la prueba de $\mathrm{t}$, de acuerdo con Wynne et al. (1970): $t=\frac{\bar{F}_{l i j}-\overline{P_{l i j}}}{\sqrt{\frac{3}{8} C M E}}$, en la que todo valor de t estimado como significativo indica que la heterosis es diferente de cero, y donde $C M E$ es el cuadrado medio del error; $3 / 8$ es una constante.

Los errores estándar de los efectos de ACG y ACE a través de localidades se estimaron como lo describió Singh (1973). La descomposición del efecto de genotipos en padres $(\mathrm{P})$, cruzas $(\mathrm{C})$ y el contraste $\mathrm{P}$ vs $\mathrm{C}$, se hizo como lo indicaron Hallauer y Miranda (1988).

\section{RESULTADOS Y DISCUSIÓN}

El análisis de varianza mostró que el efecto de localidades fue significativo $(\mathrm{P} \leq 0.01)$ en todas las variables, excepto en rendimiento total de fruto (Cuadro 1). Este comportamiento se atribuye a las diferencias edáficas, de riego y climáticas entre las dos localidades evaluadas. El efecto de genotipos fue significativo ( $\mathrm{P} \leq$ 0.01 ) en todas las variables, excepto en peso individual y diámetro de fruto. El efecto de cruzas también fue significativo $(\mathrm{P} \leq 0.01)$ en las variables evaluadas, excepto en el peso y diámetro de fruto. El efecto padres fue significativo $(P \leq 0.05)$ en rendimiento y longitud de fruto. Resultados similares reportaron Gomide et al. (2003) para rendimiento de fruto en chile dulce. El contraste $\mathrm{P} v s$. C sólo fue significativo en peso individual de fruto $(\mathrm{P} \leq 0.01)$, lo que implica existencia de heterosis.

Los cuadrados medios para la ACG fueron de mayor magnitud que los de ACE en las variables estudiadas, lo que sugiere que los efectos de la ACG contribuyen más a la variación genética de los componentes del rendimiento en las poblaciones criollas de chile 'Dulce', que los efectos de ACE. Es decir, los caracteres evaluados del germoplasma se asociaron más con efectos aditivos que con los no adictivos, como ha sido reportado en chile por Gomide et al. (2003) y Nandadevi y Hosamani (2003). Las ACG difirieron significativamente $(\mathrm{P} \leq 0.05)$ en rendimiento de fruto, número de frutos por planta, días a inicio de cosecha, longitud de fruto, y altura de planta. Para peso de fruto y diámetro de fruto no se encontró significancia.

Para ACE se encontraron efectos significativos ( $\mathrm{P} \leq$ 0.01 ) en rendimiento total de fruto, número de frutos por planta y días a inicio de cosecha, lo que indica presencia de acción genética no aditiva (dominancia) en estos caracteres. Contrariamente, las variables peso individual de fruto, altura de planta, longitud y diámetro de fruto no mostraron significancia. 
Cuadro 1. Cuadrados medios del análisis de varianza combinado para características agronómicas y rendimiento, en genotipos de chile 'Dulce' evaluadas en dos localidades de Yucatán.

\begin{tabular}{|c|c|c|c|c|c|c|c|c|}
\hline $\begin{array}{l}\text { Fuente de } \\
\text { variación }\end{array}$ & $\mathrm{gl}$ & $\begin{array}{l}\text { Rendimiento } \\
\text { de fruto } \\
\text { (g/planta) }\end{array}$ & $\begin{array}{l}\text { Peso individual } \\
\text { del fruto } \\
\text { (g) }\end{array}$ & $\begin{array}{l}\text { Núm. de } \\
\text { frutos } \\
\text { por planta }\end{array}$ & $\begin{array}{l}\text { Días a } \\
\text { cosecha }\end{array}$ & $\begin{array}{l}\text { Altura de } \\
\text { planta } \\
(\mathrm{cm})\end{array}$ & $\begin{array}{l}\text { Longitud de } \\
\text { fruto } \\
\text { (mm) }\end{array}$ & $\begin{array}{l}\text { Diámetro de } \\
\text { fruto } \\
(\mathrm{mm})\end{array}$ \\
\hline Localidad (Loc) & 1 & $1610 \mathrm{~ns}$ & $711.6^{* *}$ & $28.5 * *$ & $18021.4^{* * *}$ & $2577.2 * *$ & $641.6^{* *}$ & $3084.2 * *$ \\
\hline Genotipos (G) & 27 & $14607 * *$ & $28.5 \mathrm{~ns}$ & $14.2 * *$ & $57.4 * *$ & $59.3^{* *}$ & $31.7 * *$ & $18.8 \mathrm{~ns}$ \\
\hline Cruzas (C) & 20 & $16782 * *$ & $22.2 \mathrm{~ns}$ & $17.4^{* *}$ & $67.9 * *$ & $53.5^{*}$ & $31.9 * *$ & $18.0 \mathrm{~ns}$ \\
\hline ACG & 6 & $26866^{* *}$ & $38.3 \mathrm{~ns}$ & $35.4 * *$ & $100.5^{* *}$ & $79.2 *$ & $75.8^{* *}$ & $29.5 \mathrm{~ns}$ \\
\hline P vs. C & 1 & $14988 \mathrm{~ns}$ & $108.6^{* *}$ & $5.3 \mathrm{~ns}$ & $0.9 \mathrm{~ns}$ & $74.7 \mathrm{~ns}$ & $33.6 \mathrm{~ns}$ & $10.0 \mathrm{~ns}$ \\
\hline Loc $x \mathrm{G}$ & 27 & $15807 * *$ & $26.5 \mathrm{~ns}$ & $13.1^{* *}$ & $55.8^{* *}$ & $78.4^{* *}$ & $28.9 * *$ & $37.7 * *$ \\
\hline Loc $x$ Cruzas & 20 & $14475^{* *}$ & $25.4 \mathrm{~ns}$ & $10.3 * *$ & $54.5^{* *}$ & $74.2^{* *}$ & $29.5^{* *}$ & $41.1^{* *}$ \\
\hline Loc x ACG & 6 & $11367 *$ & $33.5 \mathrm{~ns}$ & $6.4 \mathrm{~ns}$ & $16.6 \mathrm{~ns}$ & $53.2 \mathrm{~ns}$ & $29.5 \mathrm{~ns}$ & $57.5^{* *}$ \\
\hline Loc $\mathrm{x}$ ACE & 14 & $15806^{* *}$ & $21.9 \mathrm{~ns}$ & $11.9 * *$ & $70.7 * *$ & $83.2 * *$ & $29.5 *$ & $34.0^{*}$ \\
\hline Loc $\times \mathrm{P}$ & 6 & $11489 * *$ & $32.9 \mathrm{~ns}$ & $12.2^{* *}$ & $63.2 * *$ & $104.4^{*}$ & $29.5^{*}$ & $31.6 \mathrm{~ns}$ \\
\hline
\end{tabular}

*,**, ns: significativo a 0.05, 0.01, y no significativo respectivamente. Loc = localidad; Bloques(Loc) = repetición anidado en localidad; ACG = aptitud combinatoria general; ACE = aptitud combinatoria específica; Loc x Cruza = interacción localidad x cruza; Loc x ACG = interacción localidad x ACG; Loc $\times$ ACE = interacción localidad x ACE; Loc x P = interacción localidad x padres; Loc x P vs. C $=$ interacción localidad $\mathrm{x}$ padres $v s$. cruzas; CV $=$ coeficiente de variación; gl = grados de libertad.

Estos resultados coinciden con los reportados en chile por Gomide et al. (2003), pero difieren en peso de fruto con lo reportado en chile 'Serrano' por Martínez et al. (2005), quienes encontraron que los efectos de dominancia fueron los que determinaron la respuesta de esta variable; y coinciden con los de Lippert (1975), quien encontró que los efectos aditivos fueron más importantes que los de dominancia para el peso del fruto.

La interacción localidad $\mathrm{x}$ genotipo mostró significancia $(\mathrm{P} \leq 0.01)$ en las variables evaluadas, con excepción del peso individual, lo que demuestra que los genotipos difirieron entre los ambientes de prueba. Resultados similares fueron reportados por Wani et al. (2003) para rendimiento de fruto. La interacción localidades $\mathrm{x}$ cruza fue significativa $(\mathrm{P} \leq 0.05)$, lo que indica que las cruzas respondieron también en forma diferente en los dos ambientes de evaluación. Echandi (2005) reportó resultados similares para rendimiento de fruto y tamaño de frutos en chile 'Jalapeño'. En la interacción localidades $x$ ACG hubo efectos significativos $(P \leq 0.05)$ en rendimiento total de fruto y diámetro de fruto. El efecto de localidades $x$ ACE fue significativo $(P \leq 0.05)$ en las características medidas, excepto para peso individual de frutos.

Estos resultados muestran que el ambiente influye en el rendimiento de fruto y características agronómicas del chile 'Dulce'. Al respecto, Echandi (2005) encontró estabilidad a través de localidades en chile para peso y número de frutos por planta, días a cosecha y longitud del fruto, similarmente Ben-Chaim y Paran (2000) encontraron poco efecto del ambiente en C. annuиm. Se considera que en el presente trabajo las condiciones de evaluación fueron contrastantes, ya que en Akil se aplicó riego por inundación con suelo poco profundo, mientras que en Acanceh el suelo es profundo, con mayor cantidad de materia orgánica y se usó riego por goteo, además de que en cada sitio la siembra se hizo en estación y años diferentes.

El efecto localidad $x$ padres fue significativo $(P \leq 0.05)$ en rendimiento total de fruto, número de frutos por planta, días a inicio de cosecha, altura de planta y longitud de fruto, lo que implica que el ambiente afectó el comportamiento de los padres. Según Baker (1988), en pruebas de rendimiento efectuadas en una localidad, la frecuencia y naturaleza de las interacciones debe ser apoyadas por información colectada en varios años. El efecto localidades x P vs. C también fue significativo ( $\mathrm{P} \leq$ 0.01 ) en rendimiento total de fruto y número de frutos por planta, por lo que el efecto ambiental debió haber influido en la heterosis registrada de ambas variables.

Las medias de progenitores y sus cruzas se muestran en el Cuadro 2. En rendimiento total de fruto, el progenitor $\mathrm{P} 7$ y las cruzas $\mathrm{P} 2 \times \mathrm{P} 3, \mathrm{P} 2 \times \mathrm{P} 6, \mathrm{P} 3 \times \mathrm{P} 4$ y P3 x P6 resultaron superiores a la media de los progenitores y cruzas, respectivamente. En peso individual de fruto no hubo diferencias entre padres ni entre cruzas, mientras 
que en número de frutos por planta sólo las cruzas $\mathrm{P} 2 \mathrm{x}$ P3, P2 x P6 y P3 x P4 fueron superiores al resto. Los progenitores más precoces (con menor tiempo a inicio de

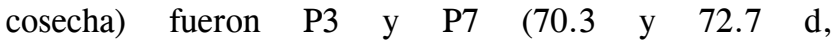
respectivamente), en tanto que P6 fue el progenitor más tardío con $77.2 \mathrm{~d}$.

Las cruzas $\mathrm{P} 2$ x P7, P3 x P5, P4 x P7 y P5 x P7 fueron los híbridos más tardíos, con más de $78 \mathrm{~d}$ al inicio de cosecha; de las cuatro cruzas más precoces ( $\mathrm{P} 3$ x $\mathrm{P} 4, \mathrm{P} 3$ x P7, P4 x P5 y P4 x P6), dos de ellas involucraron a uno de los progenitores más precoces ( $\mathrm{P} 3$ y $\mathrm{P} 7)$. El progenitor P4 fue el más alto $(52.7 \mathrm{~cm})$, y las cruzas $\mathrm{P} 2 \times \mathrm{P} 5, \mathrm{P} 2 \times$ P6 y P4 x P6 también sobresalieron en altura de planta y superaron $(\mathrm{P} \leq 0.05)$ al resto de híbridos. $\mathrm{P} 2$ fue uno de los dos genotipos de menor altura, y en dos de sus cruzas con otros progenitores (P5 y P6) manifestó el mayor vigor híbrido (fueron las más altas). Estos resultados sugieren que para este tipo de chile, una mayor altura de planta no implica necesariamente mayor rendimiento de fruto. En mejoramiento genético la altura de planta adquiere relevancia al considerar el tipo de manejo al que será sometido; así, las plantas compactas y de porte mediano son idóneas para el cultivo en campo, mientras que para el cultivo en invernaderos las plantas altas o con hábito de crecimiento indeterminado serían óptimas (Greenleaf, 1986).

El progenitor P6 produjo los frutos de mayor tamaño (longitud y diámetro), aunque P1 y P4 fueron superiores en longitud de fruto, con respecto al promedio de progenitores. En cuanto a cruzas, solamente P1 x P3 fue superior a la media general para diámetro de fruto, lo cual indica poca variación del tamaño de fruto en las cruzas.

\begin{tabular}{|c|c|c|c|c|c|c|c|}
\hline Padres y cruzas & $\begin{array}{c}\text { Rendimiento de } \\
\text { fruto } \\
\text { (g/planta) }\end{array}$ & $\begin{array}{l}\text { Peso individual } \\
\text { del fruto } \\
\text { (g) }\end{array}$ & $\begin{array}{l}\text { Núm. de } \\
\text { frutos por } \\
\text { planta }\end{array}$ & $\begin{array}{l}\text { Días a inicio } \\
\text { de cosecha }\end{array}$ & $\begin{array}{l}\text { Altura de } \\
\text { planta } \\
(\mathrm{cm})\end{array}$ & $\begin{array}{l}\text { Longitud de } \\
\text { fruto } \\
\text { (mm) }\end{array}$ & $\begin{array}{l}\text { Diámetro de } \\
\text { fruto } \\
(\mathrm{mm})\end{array}$ \\
\hline P1 & 262.0 & 32.6 & 7.8 & 75.0 & 47.7 & $56.6^{\ddagger}$ & 56.5 \\
\hline P2 & 201.3 & 30.3 & 6.6 & 75.0 & 43.0 & 52.5 & 56.7 \\
\hline P3 & 239.1 & 29.7 & 8.0 & 70.3 & 43.5 & 52.9 & 57.4 \\
\hline P4 & 259.7 & 35.8 & 7.3 & 75.0 & $52.7 *$ & $58.6^{\ddagger}$ & 55.7 \\
\hline P5 & 198.0 & 34.1 & 5.8 & 76.2 & 47.5 & 55.3 & 58.7 \\
\hline P6 & 256.0 & 32.7 & 8.0 & 77.2 ‡ & 48.0 & 57.0 \# & 61.6 * \\
\hline P7 & $295.2 *$ & 35.9 & 8.3 & 72.7 & 43.0 & 54.2 & 58.0 \\
\hline $\mathrm{P} 1 \mathrm{xP} 2$ & 233.0 & 34.0 & 6.8 & 78.3 & 44.8 & 54.6 & 59.6 \\
\hline $\mathrm{P} 1 \mathrm{xP} 3$ & 268.3 & 33.1 & 8.1 & 76.2 & 49.2 & 52.1 & $62.3^{*}$ \\
\hline $\mathrm{P} 1 \mathrm{xP} 4$ & 247.3 & 37.2 & 6.8 & 72.7 & 46.2 & 57.6 & 59.5 \\
\hline P1xP5 & 236.0 & 36.4 & 6.7 & 72.7 & 47.7 & 51.7 & 61.0 \\
\hline P1xP6 & 204.0 & 35.7 & 5.8 & 76.3 & 49.3 & 57.1 & 57.3 \\
\hline $\mathrm{P} 1 \mathrm{xP7}$ & 290.4 & 37.7 & 7.5 & 75.0 & 48.8 & 53.7 & 59.1 \\
\hline $\mathrm{P} 2 \mathrm{xP} 3$ & $406.2^{*}$ & 33.5 & $12.2 \ddagger$ & 72.5 & 48.2 & 52.4 & 57.1 \\
\hline $\mathrm{P} 2 \mathrm{xP} 4$ & 284.7 & 33.3 & 8.4 & 73.8 & 46.12 & 57.2 & 60.6 \\
\hline $\mathrm{P} 2 \mathrm{xP5}$ & 228.6 & 36.6 & 6.3 & 77.2 & $52.8 \div$ & 57.5 & 59.7 \\
\hline $\mathrm{P} 2 \mathrm{xP} 6$ & $335.3^{\ddagger}$ & 35.0 & $9.6 \div$ & 73.0 & 53.5 † & 55.6 & 58.6 \\
\hline $\mathrm{P} 2 \mathrm{xP7}$ & 214.5 & 38.2 & 6.1 & $78.5 \div$ & 46.0 & 55.4 & 59.5 \\
\hline $\mathrm{P} 3 \mathrm{xP} 4$ & $360.4^{\ddagger}$ & 32.0 & $11.6^{\ddagger}$ & 70.0 & 47.0 & 50.4 & 56.6 \\
\hline $\mathrm{P} 3 \times \mathrm{P} 5$ & 198.3 & 32.1 & 6.3 & $78.5^{\ddagger}$ & 43.5 & 49.8 & 58.0 \\
\hline P3xP6 & $323.6 \neq$ & 35.3 & 9.4 & 68.0 & 51.7 & 53.2 & 58.3 \\
\hline $\mathrm{P} 3 \mathrm{xP7}$ & 248.4 & 31.6 & 7.9 & 71.5 & 44.8 & 52.0 & 57.3 \\
\hline $\mathrm{P} 4 \mathrm{xP5}$ & 251.0 & 37.0 & 7.0 & 71.5 & 45.7 & 54.1 & 55.5 \\
\hline $\mathrm{P} 4 \mathrm{xP} 6$ & 272.5 & 34.0 & 8.2 & 71.5 & $53.7 *$ & 55.9 & 58.0 \\
\hline P4xP7 & 279.6 & 33.6 & 8.3 & $80.7 \ddagger$ & 44.0 & 56.56 & 55.7 \\
\hline P5xP6 & 237.2 & 35.4 & 6.8 & 75.0 & 47.7 & 53.7 & 57.3 \\
\hline P5xP7 & 228.0 & 35.2 & 6.6 & $79.5 *$ & 48.5 & 54.5 & 57.6 \\
\hline P6xP7 & 244.6 & 35.8 & 7.9 & 75.3 & 49.2 & 55.0 & 57.3 \\
\hline Mg & 260.8 & 34.4 & 7.7 & 74.6 & 47.6 & 54.5 & 58.2 \\
\hline Mp & 244.5 & 33.0 & 7.4 & 74.4 & 46.5 & 55.3 & $\mathbf{5 7 . 8}$ \\
\hline Mc & 266.3 & 34.9 & 7.8 & $\mathbf{7 4 . 7}$ & 48.0 & 54.2 & 58.4 \\
\hline
\end{tabular}

$\mathrm{Mg}=$ media general; $\mathrm{Mp}=$ media de padres; $\mathrm{Mc}=$ media de cruzas. ${ }^{\ddagger}$ Mayor que $\mu+2 \sigma$. 


\begin{tabular}{|c|c|c|c|c|c|c|c|c|c|c|c|c|c|c|}
\hline \multirow{3}{*}{$\begin{array}{l}\text { Padre } \\
\text { o } \\
\text { cruza }\end{array}$} & \multicolumn{2}{|c|}{ Rendimiento } & \multicolumn{2}{|c|}{ Peso de fruto } & \multicolumn{2}{|c|}{ Núm. frutos/planta } & \multicolumn{2}{|c|}{ Días a cosecha } & \multicolumn{2}{|c|}{ Altura de planta } & \multicolumn{2}{|c|}{ Longitud de fruto } & \multicolumn{2}{|c|}{ Diámetro de frutc } \\
\hline & ACG & & ACG & & ACG & & ACG & & ACG & & ACG & & ACG & \\
\hline & $\begin{array}{c}\mathrm{o} \\
\mathrm{ACE}\end{array}$ & $\begin{array}{c}\mathrm{H} \\
(\%)\end{array}$ & $\begin{array}{c}\mathrm{o} \\
\mathrm{ACE}\end{array}$ & $\begin{array}{c}\mathrm{H} \\
(\%)\end{array}$ & $\begin{array}{c}\mathrm{o} \\
\mathrm{ACE}\end{array}$ & $\begin{array}{c}\mathrm{H} \\
(\%)\end{array}$ & $\stackrel{\mathrm{o}}{\mathrm{ACE}}$ & $\begin{array}{c}\mathrm{H} \\
(\%)\end{array}$ & $\stackrel{\mathrm{o}}{\mathrm{ACE}}$ & $\begin{array}{c}\mathrm{H} \\
(\%)\end{array}$ & $\stackrel{\mathrm{o}}{\mathrm{ACE}}$ & $\begin{array}{c}\mathrm{H} \\
(\%)\end{array}$ & $\stackrel{0}{\mathrm{ACE}}$ & $\begin{array}{c}\mathrm{H} \\
(\%)\end{array}$ \\
\hline P1 & -23.7 & & 1.0 & & -1.0 & & 0.7 & & -0.4 & & 0.2 & & 1.7 & \\
\hline $\mathrm{P} 2$ & 20.9 & & 0.2 & & 0.5 & & 1.1 & & 0.7 & & 1.4 & & 1.0 & \\
\hline P3 & 41.5 & & -2.4 & & 1.7 & & -2.2 & & -0.8 & & -3.2 & & -0.1 & \\
\hline P4 & 19.6 & & -0.4 & & 0.7 & & -1.5 & & -1.1 & & 1.2 & & -0.9 & \\
\hline P5 & -43.7 & & 0.7 & & -1.4 & & 1.3 & & -0.5 & & -0.9 & & -0.2 & \\
\hline P6 & 3.9 & & 0.4 & & 0.1 & & -1.7 & & 3.4 & & 1.0 & & -0.7 & \\
\hline P7 & -18.4 & & 0.6 & & -0.5 & & 2.5 & & -1.4 & & 0.3 & & -0.7 & \\
\hline $\mathrm{P} 1 \mathrm{xP} 2$ & -30.4 & 0.6 & -2.1 & 8.2 & -0.5 & -5.5 & 1.9 & 4.4 & -3.4 & -1.1 & -1.3 & 0.0 & -1.5 & 5.3 \\
\hline P1xP3 & -15.8 & 7.1 & -0.4 & 6.2 & -0.3 & 3.0 & 3.1 & 4.8 & 2.3 & 7.9 & 0.7 & -4.9 & 2.4 & $9.6^{*}$ \\
\hline P1xP4 & -14.8 & -5.2 & 1.8 & 8.6 & -0.7 & -10.5 & -1.1 & -3.1 & -0.3 & -8.0 & 1.9 & 0.0 & 0.3 & 6.0 \\
\hline P1xP5 & 37.2 & 2.6 & -0.1 & 9.1 & 1.3 & -2.5 & -3.9 & -3.9 & 0.5 & 0.2 & -1.9 & -7.6 & 1.1 & 5.8 \\
\hline P1xP6 & -42.4 & -21.2 & -0.5 & 9.2 & -1.1 & -27.4 & 2.8 & 0.3 & -1.6 & 3.1 & 1.6 & 0.5 & -2.1 & -3.0 \\
\hline P1xP7 & 66.3 & 4.2 & 1.3 & 10.0 & 1.3 & -6.8 & -2.8 & 1.6 & 2.6 & 7.7 & -1.1 & -3.1 & -0.2 & 3.2 \\
\hline P2xP3 & 77.5 & $84.5^{* *}$ & 0.7 & 11.6 & 2.2 & $67.4^{* *}$ & -1.0 & -0.2 & 0.2 & 11.4 & -0.1 & -0.6 & -2.1 & 0.1 \\
\hline $\mathrm{P} 2 \mathrm{xP} 4$ & -22.1 & 23.5 & -1.4 & 0.7 & -0.6 & 21.2 & -0.4 & -1.6 & -1.4 & -3.5 & 0.3 & 3.0 & 2.1 & 7.7 \\
\hline P2xP5 & -14.9 & 14.5 & 0.8 & 13.5 & -0.6 & 1.1 & 0.1 & 2.1 & 4.6 & $16.8^{*}$ & 2.7 & 6.7 & 0.6 & 3.3 \\
\hline P2xP6 & 44.2 & $46.7^{* *}$ & -0.5 & 11.0 & 1.2 & $31.9 *$ & -1.0 & -4.1 & 1.4 & $17.6^{*}$ & -1.0 & 1.6 & -0.1 & -1.0 \\
\hline P2xP7 & -54.3 & -13.6 & 2.5 & 15.5 & -1.7 & -18.1 & 0.2 & 6.3 & -1.3 & 7.0 & -0.6 & 3.8 & 0.9 & 3.8 \\
\hline $\mathrm{P} 3 \mathrm{xP} 4$ & 33.0 & $44.5^{* *}$ & -0.1 & -2.3 & 1.4 & $51.2^{* *}$ & -0.9 & -3.7 & 0.8 & -2.3 & -2.0 & $-9.7 *$ & -0.8 & 0.1 \\
\hline P3xP5 & -65.7 & -9.2 & -1.1 & 0.4 & -1.8 & -8.5 & 4.8 & 7.2 & -3.3 & -4.4 & -0.4 & -7.9 & 0.0 & -0.1 \\
\hline P3xP6 & 11.9 & $30.8^{*}$ & 2.4 & 13.0 & -0.3 & 16.7 & -2.7 & $-7.8^{*}$ & 1.0 & 12.9 & 1.1 & -3.2 & 0.7 & -2.0 \\
\hline P3xP7 & -40.9 & -7.0 & -1.5 & -3.8 & -1.1 & -3.0 & -3.4 & 0.0 & -1.1 & 3.7 & 0.6 & -2.9 & -0.2 & -0.7 \\
\hline P4xP5 & 8.9 & 9.7 & 1.9 & 5.7 & -0.0 & 6.4 & -2.9 & -5.4 & -0.8 & -8.8 & -0.5 & -5.1 & -1.7 & -3.0 \\
\hline P4xP6 & -17.2 & 5.7 & -0.8 & -0.7 & -0.4 & 6.7 & 0.1 & $-6.0^{*}$ & 3.4 & 6.6 & -0.6 & -3.4 & 1.2 & -1.2 \\
\hline P4xP7 & 12.2 & 0.8 & -1.4 & -6.4 & 0.3 & 6.6 & 5.0 & $9.3^{*}$ & -1.6 & -8.0 & 0.8 & 0.3 & -1.0 & -2.0 \\
\hline P5xP6 & 10.7 & 4.5 & -0.5 & 6.0 & 0.3 & -2.5 & 0.8 & -2.2 & -3.3 & -0.2 & -0.7 & -4.4 & -0.1 & -4.8 \\
\hline P5xP7 & 23.9 & -7.5 & -0.9 & 0.4 & 0.8 & -6.2 & 1.0 & 6.8 & 2.3 & 7.2 & 0.8 & -0.6 & 0.2 & -1.3 \\
\hline P6xP7 & -7.2 & -11.3 & 0.0 & 4.5 & 0.4 & -3.6 & -0.1 & 0.6 & -0.9 & 8.1 & -0.5 & -1.2 & 0.3 & -4.2 \\
\hline ES gi-gj & 8.8 & & 0.6 & & 0.3 & & 0.6 & & 0.7 & & 0.5 & & 0.6 & \\
\hline ES sij-sjj & 25.6 & & 1.7 & & 0.8 & & 1.7 & & 2.0 & & 1.4 & & 1.6 & \\
\hline
\end{tabular}

** significativo $(\mathrm{P} \leq 0.01)$, * significativo $(\mathrm{P} \leq 0.05)$. ACG se refiere a progenitores, y ACE a las cruzas; ES gi-gj = error estándar de una diferencia de medias de padres; $\mathrm{H}=$ heterosis; ES sij-sij = error estándar de los efectos de ACE. 
Los valores estimados de las aptitudes combinatorias general (ACG) y específica (ACE) se presentan en el Cuadro 3. Para ACG en rendimiento total de fruto, los progenitores P2, P3 y P4 presentaron los valores más altos y positivos, con 20.9, 41.5 y 19.6 g/planta, respectivamente. Según Zewdie y Bosland (2000), los valores positivos de ACG pueden ser interpretados como una manifestación de la variabilidad presente en los progenitores, que puede ser trasmitida a su progenie. Al estudiar el contenido de capsaicina en los progenitores de Capsicum pubescens $R$. \& $P$., esos mismos autores encontraron que los progenitores con valores negativos de ACG trasmitieron a sus cruzas una baja capacidad para producir capsaicina. Según Lippert (1975) y Zewdie et al. (2000), con base en la ACG de los padres se puede predecir la contribución que cada uno de ellos hará a su progenie. Esto permitiría seleccionar plantas que combinen las características superiores de los progenitores, así como predecir las cruzas con mayor potencial. Por su parte, Ahmed et al. (1997) y Zewdie y Bosland (2000) señalaron que con buenos valores de ACG y ACE de padres y sus cruzas, se pueden definir los métodos de mejoramiento más apropiados para aprovechar alelos favorables.

Respecto a los efectos de aptitud combinatoria específica (Cuadro 3), para rendimiento de fruto las mejores cruzas fueron P2 x P3, P1 x P7, P2 x P6, P1 x P5 y P3 x P4, con valores de 77.5, 66.3, 44.2, 37.2 y $33.0 \mathrm{~g} /$ planta, respectivamente. Las cruzas P2 x P3, P2 x P6 y P3 x P4 presentaron los valores más altos y positivos de heterosis, las cuales fueron diferentes de cero ( $\mathrm{P} \leq$ 0.01) para rendimiento, mientras que las cruzas con efectos negativos fueron P3 x P5 (-65.7\%), P2 x P7 $(-54.3 \%)$, P1 x P6 (-42.4\%) y P3 x P7 (-40.9\%). Kumar y Lal (2001) encontraron que la heterosis varió para rendimiento de fruto fresco por planta, longitud de fruto y altura de planta. Según Han et al. (1991), los valores bajos o negativos de heterosis podrían deberse a insuficiente diversidad genética entre las poblaciones y a mayor variabilidad genética dentro de poblaciones.

Se puede afirmar entonces que no se puede generalizar que al cruzar un progenitor de bajo valor de ACG con otro de alto efecto de ACG se obtendrá un híbrido con una buena respuesta de ACE, pero se puede afirmar que si el efecto de ACG de un progenitor es negativo la ACE de sus cruzas será negativa, o positiva de magnitud pequeña. También se infiere que al cruzar entre sí a progenitores con buena ACG, la progenie manifiesta una ACE alta (por ejemplo P3 x P4), aunque algunas cruzas entre un progenitor con alto valor de ACG y uno de mediana ACG puede combinar mejor y producir híbridos con efectos más altos de ACE; tal es el caso de P2 x P3, que fue la cruza con la mayor ACE (77.5) y con el mayor efecto de heterosis $(84.5 \%)$.

Las variables rendimiento de fruto, número de frutos por planta y peso individual del fruto, mostraron los efectos de heterosis más altos (Cuadro 3). Por tanto, estos caracteres podrían ser mejorados más eficientemente mediante métodos de mejoramiento genético que exploten los efectos de acción génica no aditiva, como la hibridación. Para días a inicio de cosecha, altura de planta, longitud y diámetro de fruto, los valores de heterosis fueron pequeños, por lo que para estas variables se podría aplicar métodos de endocría y selección para su mejoramiento (Martínez et al., 2005).

\section{CONCLUSIONES}

Las poblaciones progenitoras $\mathrm{P} 3, \mathrm{P} 4$ y $\mathrm{P} 2$ de chile 'Dulce' sobresalieron por tener valores positivos y altos de ACG. P2 y P3 estuvieron involucrados en las mejores cuatro cruzas $(\mathrm{P} 2 \times \mathrm{P} 3, \mathrm{P} 3 \times \mathrm{P} 4, \mathrm{P} 2 \times \mathrm{P} 6$ y $\mathrm{P} 3$ x P6) para rendimiento de fruto y número de frutos por planta, con altos efectos de ACE y heterosis. En estas características la hibridación seria el método de mejoramiento más indicado. En cambio, longitud y diámetro de fruto (tamaño de fruto), altura de planta, peso de fruto y días a inicio de cosecha, podrían ser mejoradas por métodos de endocría y selección.

\section{BIBLIOGRAFÍA}

Ahmed N, S H Khan, M I Tanki (1997) Combining ability analysis for fruit yield and its component characters in sweet pepper (Capsicum annuum L.). Capsicum Eggplant Newslet. 16:27-75.

Antuna G O, F Rincón S, E Gutíerrez del R, N A Ruíz T, L Bustamante G (2003) Componentes genéticos de caracteres agronómicos y de calidad fisiológica de semillas de líneas de maíz. Rev. Fitotec. Mex. 26:11-17.

Baker R J (1988) Analysis of genotype-environmental interactions in crops. Anim. Plant Sci. 1:1-4.

Ben-Chaim A, I Paran (2000) Genetic analysis of quantitative traits in pepper (Capsicum annuum). J. Amer. Soc. Hort. Sci.125:6670.

Blank A F, W R Maluf (1997) Gene action and early testing for combining ability in sweet peppers (Capsicum annuum L.). J. Genet. Breed. 51:319-324.

Castañón N G, L Latournerie M, M Mendoza E (2005) Macro de SAS-IML para analizar los diseños II y IV de Griffing. Univ. Ciencia 21:27-35.

Cruz-Pérez A B, V A González-Hernández, R M Soto-Hernández, M A Gutiérrez-Espinosa, A A Gardea-Béjar, M Pérez-Grajales (2007) Capsaicinoides, vitamina $\mathrm{C}$ y heterosis durante el desarrollo del fruto de chile manzano. Agrociencia 41:627-635.

De Souza J A, W R Maluf (2003) Diallel analysis and estimation of genetic parameters of hot pepper (Capsicum chinense Jacq.). Sci. Agric. 60:105-113.

Duch G J (1988) La Conformación Territorial del Estado de Yucatán. Los Componentes del Medio Físico. Universidad Autónoma de Chapingo. Centro Regional de la Península de Yucatán. 427 p. 
Echandi G C R (2005) Estabilidad fenotípica del rendimiento y adaptación en líneas de chile jalapeño (Capsicum anпиит L.) durante la época lluviosa en Costa Rica. Agron. Costarricense 29: $27-44$

Falconer D S (1996) Introducción a la Genética Cuantitativa. 4a ed. Ed. ACRIBIA. Zaragoza, España. 469 p.

Gomide M L, W R Maluf, A A Gomes L (2003) Heterose e capacidade combinatória de linhagens de pimenāo (Capsicum annumm L.). Ciênc. Agrotec. Lavras 27:1007-1015.

Greenleaf H W (1986) Pepper breeding. In: Breeding Vegetable Crops. M J Basset (ed). AVI Publishing Company. University of Florida. Gainesville, Florida, USA. pp:67-134.

Griffing B (1956) Concept of general and specific combining ability in relation to diallel crossing systems. Aust. J. Biol. Sci. 9:436493.

Hallauer A R, J B Miranda F (1988) Quantitative Genetics in Maize Breeding. 2nd ed. Iowa State University Press. Ames, Iowa. pp:60-62.

Han G C, S K Vasal, D L Beck, E Elias (1991) Combining ability of inbred lines derived from CIMMYT maize (Zea mays L.) germoplasm. Maydica 36:57-67.

Jinks J L (1954) The analysis of continuous variation in a diallel cross of Nicotiana rustica varieties. Genetics 39:767-788.

Jinks J L, B I Hayman (1953) The analysis of diallel crosses. Maize Genet. Coop. Newslet. 27:48-54.

Kumar R, G Lal (2001) Expresion of heterosis in hot pepper (Capsicum annuum L.). Capsicum Eggplant Newslet. 20:38-41.

Latournerie M L, J L Chávez S, M Pérez P, C F Hernández C, R Martínez V, L M Arias R, G Castañón N (2001) Exploración de la diversidad morfológica de chiles regionales en Yaxcabá, Yucatán, México. Agron. Mesoam. 12:41-47.

Lippert L F (1975) Heterosis and combining ability in chili peppers by diallel analysis. Crop Sci. 15:323-325.

Martínez Z G, J R A Dorantes G, M Ramírez M, A de la Rosa L, O Pozo C (2005) Efectos genéticos y heterosis en la vida de anaquel del chile serrano. Rev. Fitotec. Mex. 28:327-332.

Milerue N, M Nikornpun (2000) Studies on heterosis of chili (Capsicum annuum L.). Kasetsart J. (Nat. Sci.) 34:190-196.

Mishra R S, R E Lotha R, S N Mishra (1989) Heterosis in chilli by diallel analysis. South Indian Hort. 37:179-180.

Nandadevi N, R M Hosamani (2003) Estimation of heterosis, combining ability and per se performance in summer grown chilli (Capsicum annumm L.) for yield and resistance to leaf curl complex. Capsicum Eggplant Newslet. 22:59-62.
Pérez-Grajales M, V A González-Hernández, A Peña-Lomelí, J Sahagún-Castellanos (2009) Combining ability and heterosis for fruit yiled and quality in manzano hot pepper (Capsicum pubescens R \& P) landraces. Rev. Chapingo S. Hort. 15:47-55.

Pozo C O, S Montes H, E Redondo J (1991) Chile (Capsicum spp.). In: Avances en el Estudio de los Recursos Fitogenéticos de México. Sociedad Mexicana de Fitogenética. R Ortega P, G Palomino H, F Castillo G, V A González H, M Livera M (eds). SOMEFI. Chapingo, Texcoco, Estado de México, México. pp:217-238.

SAGARPA, Secretaría de Agricultura, Ganadería, Desarrollo Rural, Pesca y Alimentación (2008) Anuario Estadístico de la Producción Agrícola a Nivel Estatal, Distrital y Municipal de Yucatán 2006. Secretaría de Agricultura, Ganadería, Desarrollo Rural, Pesca y Alimentación. 328 p.

Seneviratne K G S, K N Kannangara (2004) Heterosis, heterobeltiosis and commercial heterosis for agronomic traits and yield of Chilli (Capsicum annumm L.). Ann. Sri Lanka Depar. Agic. 6:195-201

Singh D (1973) Diallel analysis for combining ability over several environments-II. Indian J. Genet. \& Plant Breed. 33:469-481.

Soria $\mathbf{F}$ M de J, J M Tun S, J A Trejo $R$, y $R$ Terán $S$ (2000) Tecnología para la Producción de Hortalizas a Cielo Abierto en la Península de Yucatán. 3er. ed. Instituto Tecnológico Agropecuario No. 2. Conkal, Yucatán. 430 p.

Sprague G F, L A Tatum (1942) General vs specific combining ability in single crosses of corn. J. Amer. Soc. Agron. 34:923-932.

Wani K P, N Ahmed, M I Tanki, R Narayan (2003) Stability analysis for yield and quality characters in hot pepper (Capsicum annumm L.) for yield and resistance to leaf curl complex. Capsicum and Eggplant Newslet. 22:75-78.

Wynne J C, D A Emery, P W Rice (1970) Combining ability estimates in Arachis hypogaea L. II. Field performance of $\mathrm{F}_{1}$ hybrids. Crop Sci. 10:713-715.

Yan W, L A Hunt (2002) Biplot analysis of diallel data. Crop Sci. 42:21-30.

Zewdie Y, P W Bosland (2000) Evaluation of genotype, environment, and genotype-by-environment interaction for capsaicinoids in Capsicum Annuum L. Euphytica 111:185-190.

Zewdie Y, P W Bosland, R Steiner (2000) Combining ability and heterosis for capsaicinoids in Capsicum pubescens. HortScience $36: 1315-1317$ 